\title{
Investigation of State Transition Phenomena in Cross-Coupled Chaotic Circuits
}

\author{
Yumiko Uchitani and Yoshifumi Nishio \\ Department of Electrical and Electronic Engineering, Tokushima University \\ 2-1 Minami-Josanjima, Tokushima, 770-8506 JAPAN \\ Email: \{uchitani,nishio\}@ee.tokushima-u.ac.jp
}

\begin{abstract}
Studies on chaos synchronization in coupled chaotic circuits are extensively carried out in various fields. In this study, two simple chaotic circuits cross-coupled by inductors are investigated. Interesting state transition phenomenon around chaos synchronization is observed by computer simulations and circuit experiments.
\end{abstract}

\section{INTRODUCTION}

Synchronization phenomena in complex systems are very good models to describe various higher-dimensional nonlinear phenomena in the field of natural science. Studies on synchronization phenomena of coupled chaotic circuits are extensively carried out in various fields [1]-[9]. We consider that it is very important to investigate the phenomena related with chaos synchronization to realize future engineering application utilizing chaos. We have reported an interesting state transition phenomenon observed in simple coupled chaotic circuits [10].

In this study, we investigate the state transition phenomena in detail by computer simulations and circuit experiments.

\section{Circuit Model}

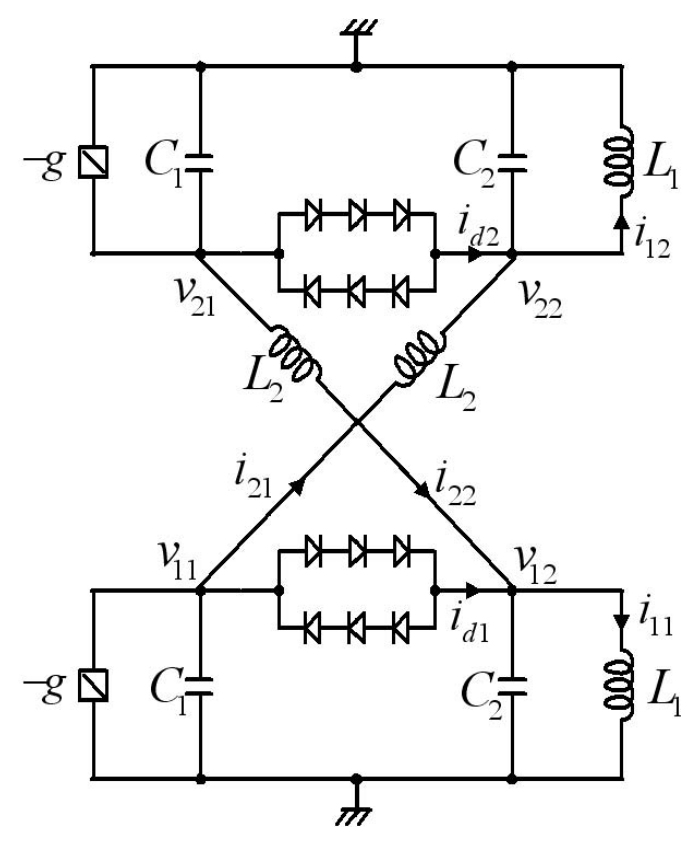

Fig. 1. Circuit model.
Figure 1 shows the circuit model [10]. In the circuit, two Shinriki-Mori chaotic circuits [11][12] are cross-coupled via inductors $L_{2}$.

By using the following variables and the parameters,

$$
\left\{\begin{array}{l}
x_{1}=\sqrt{\frac{L_{1}}{C_{2}}} \frac{i_{11}}{V}, \quad y_{1}=\frac{v_{11}}{V}, \quad z_{1}=\frac{v_{12}}{V}, \\
x_{2}=\sqrt{\frac{L_{1}}{C_{2}}} \frac{i_{21}}{V}, \quad y_{2}=\frac{v_{21}}{V}, \quad z_{2}=\frac{v_{22}}{V}, \\
w_{1}=\sqrt{\frac{L_{1}}{C_{2}}} \frac{i_{12}}{V}, \quad w_{2}=\sqrt{\frac{L_{1}}{C_{2}}} \frac{i_{22}}{V}, \\
\alpha=\frac{C_{2}}{C_{1}}, \quad \beta=\sqrt{\frac{L_{1}}{C_{2}}} G, \quad \gamma=\sqrt{\frac{L_{1}}{C_{2}}} g, \\
\delta=\frac{L_{1}}{L_{2}}, \quad t=\sqrt{L_{1} C_{2}} \tau,
\end{array}\right.
$$

the normalized circuit equations are given as follows.

$$
\left\{\begin{array}{l}
\dot{x}_{1}=z_{1} \\
\dot{x}_{2}=z_{2} \\
\dot{y}_{1}=\alpha\left\{\gamma y_{1}-w_{1}-\beta f\left(y_{1}-z_{1}\right)\right\} \\
\dot{y}_{2}=\alpha\left\{\gamma y_{2}-w_{2}-\beta f\left(y_{2}-z_{2}\right)\right\} \\
\dot{z}_{1}=\beta f\left(y_{1}-z_{1}\right)+w_{2}-x_{1} \\
\dot{z}_{2}=\beta f\left(y_{2}-z_{2}\right)+w_{1}-x_{2} \\
\dot{w}_{1}=\delta\left(y_{1}-z_{2}\right) \\
\dot{w}_{2}=\delta\left(y_{2}-z_{1}\right)
\end{array}\right.
$$

where $f$ are the nonlinear functions corresponding to the $v-$ $i$ characteristics of the nonlinear resistors consisting of the diodes and are assumed to be described by the following 3segment piecewise-linear functions:

$$
\begin{aligned}
& f\left(y_{1}-z_{1}\right)= \begin{cases}y_{1}-z_{1}-1 & \left(y_{1}-z_{1}>1\right) \\
0 & \left(\left|y_{1}-z_{1}\right| \leq 1\right) \\
y_{1}-z_{1}+1 & \left(y_{1}-z_{1}<-1\right)\end{cases} \\
& f\left(y_{2}-z_{2}\right)= \begin{cases}y_{2}-z_{2}-1 & \left(y_{2}-z_{2}>1\right) \\
0 & \left(\left|y_{2}-z_{2}\right| \leq 1\right) \\
y_{2}-z_{2}+1 & \left(y_{2}-z_{2}<-1\right) .\end{cases}
\end{aligned}
$$




\section{State Transition Phenomenon}

From the circuit in Fig. 1, we could observe interesting state transition phenomenon [10]. An example of the phenomenon is shown in Figs. 2 and 3. Figure 2 shows computer calculated results of Eq. (2) with the Runge-Kutta method. While, Fig. 3 shows the corresponding circuit experimental result. The two circuits exhibited chaos but almost synchronized in in-phase in the sense that the attractor was almost in the quadrant I or III on the $y_{1}-y_{2}$ plane. The behaviors of the circuits are very interesting because the solutions on the $y_{i}-z_{i}$ planes seem to be attracted to the fixed points located at around $\left(y_{i}, z_{i}\right)=( \pm 1.2,0)$. However, after converging to the fixed points the solution abruptly moves toward the other fixed point. When one circuit switches to/from the positive region from/to the negative region in this way, the other follows the transition after a few instants.
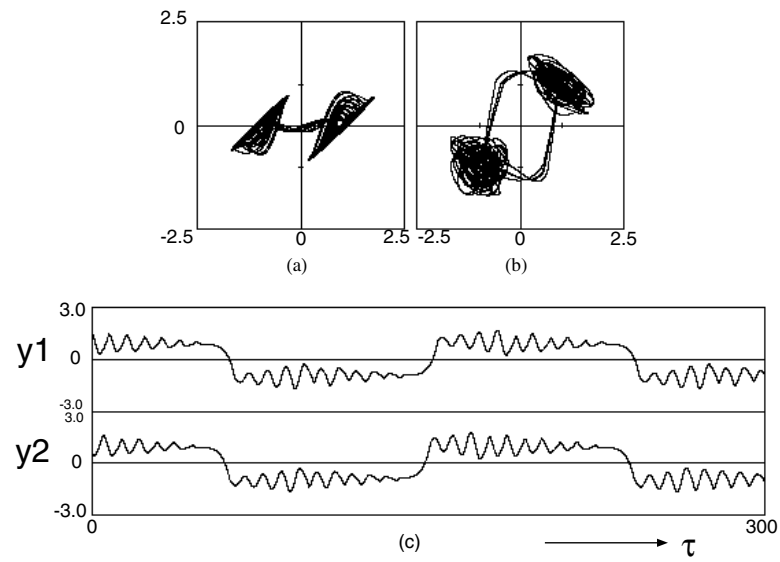

Fig. 2. State transition phenomenon around in-phase synchronization (computer calculated result). $\alpha=1.5, \beta=5.0, \gamma=0.2$, and $\delta=0.005$ (a) Attractor on $y_{1}-z_{1}$ plane. (b) Attractor on $y_{1}-y_{2}$ plane. (c) Time waveform.

\section{Detailed investigation}

In this study, we investigate the above-mentioned state transition phenomena in detail. First, we vary the coupling parameter $\delta$ and observe the phenomena. The results are shown in Fig. 4. We can see that the sojourn time between the transitions becomes shorter as increasing the coupling parameter $\delta$. The corresponding circuit experimental results are shown in Fig. 5. We can say that this interesting phenomenon can be observed from both computer calculations and circuit experiments.

Next, Fig. 6 shows how the variables change around the transitions. The variables $x$ and $z$ correspond to the current and the voltage of the LC resonator in the original chaotic circuit. Hence, they oscillate around zero and its amplitude decays as time goes. On the other hand, the variable $y$ corresponds to the voltage across the capacitor in parallel with the negative resistor and the value tends to go toward the equilibrium points located around \pm 1.2 .

When the amplitude of the oscillation of the LC resonator approaches to zero, the state transition occurs; namely $y$ abruptly moves toward to the other fixed point quickly. Though

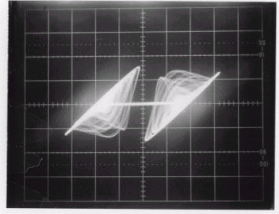

(a)

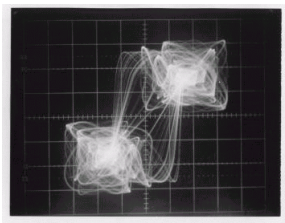

(b)

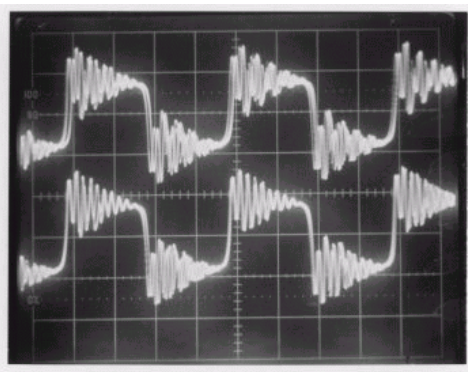

(c)

Fig. 3. State transition phenomenon around in-phase synchronization (circuit experimental result). $L_{1}=9.93 \mathrm{mH}, L_{2}=800 \mathrm{mH}, C_{1}=32.8 \mathrm{nF}$, and $C_{2}=49.5 \mathrm{nF}$, and $g=683 \mathrm{mS}$. (a) Attractor on $v_{11}-v_{12}$ plane. Horizontal and vertical: 1 V/div. (b) Attractor on $v_{11}-v_{21}$ plane. Horizontal and vertical: 1 V/div. (c) Time waveform $v_{11}$ and $v_{21}$. Horizontal $1.0 \mathrm{~ms} / \mathrm{div}$ and vertical: $2 \mathrm{~V} /$ div.

the mechanism of this transition cannot be explained very well, we consider that this is because the coupling inductor can be regarded as a short circuit when the oscillation stops. In other words, the coupling inductor plays a role of a kind of switch depending on the oscillation amplitude of the LC resonator.

Moreover, Fig. 7 shows the magnification of the time waveform of $y$. We can see that the switching timing of $y_{1}$ and $y_{2}$ are synchronized in in-phase, however, small oscillations between the transitions are synchronized in anti-phase.

\section{ANTI-PHASE STATE TRANSITION}

Next, similar state transition can be observed around antiphase synchronization as shown in Figs. 8 and 9. Similar to the in-phase, the sojourn time between the transitions decreases as the coupling $\delta$ increases.

\section{QUADRATURE-PHASE STATE TRANSITION}

We also observed an interesting state transition around quadrature-phase, namely 90 degrees. The computer calculated results are shown in Fig. 10 and the corresponding circuit experimental results are shown in Fig. 11. In this state, the solution moves to the quadrant I, II, III, and IV on the $y_{1}-y_{2}$ plane in order as shown in the figures.

\section{CONCLUSIONS}

In this study, we have investigated interesting state transition phenomenon observed from two Shinriki-Mori chaotic circuits cross-coupled by inductors.

Investigating the coexistence of the states and statistical analysis of the observed phenomena are our important future work as well as more detailed explanation of the mechanism of their generations. 

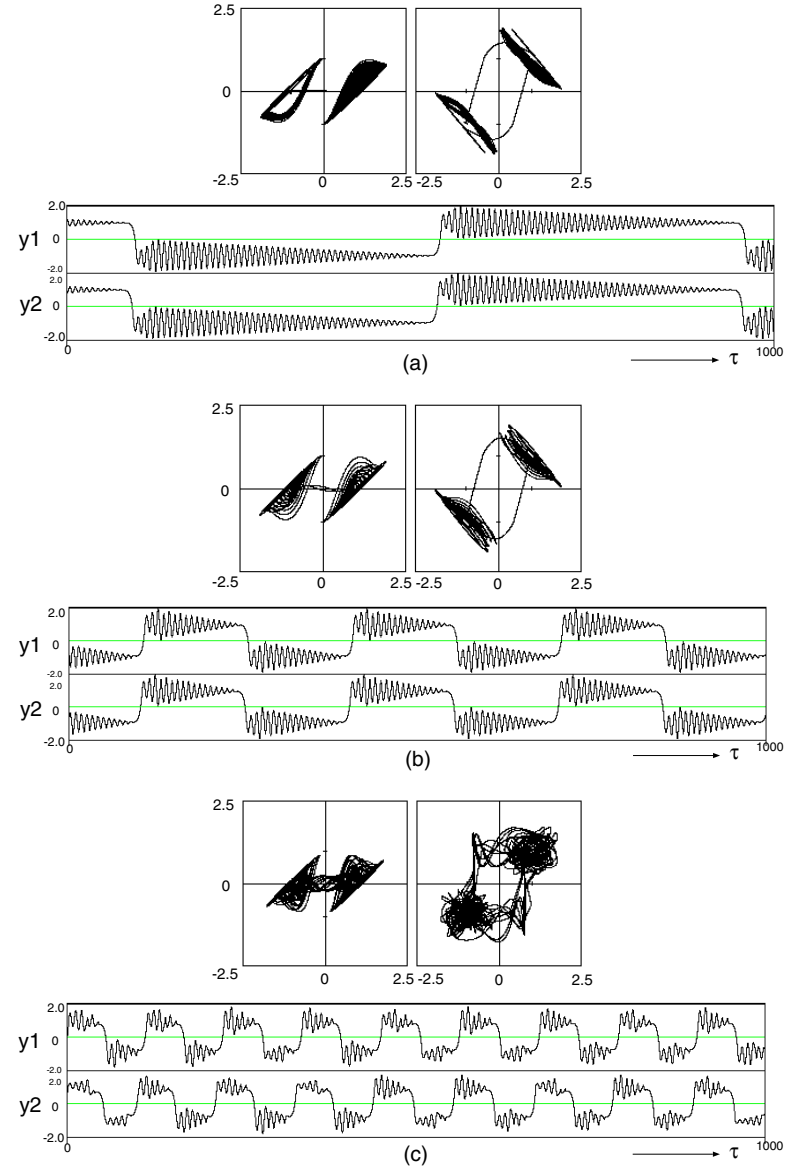

Fig. 4. State transition phenomenon for different coupling parameters (computer calculated result). $\alpha=1.5, \beta=5.0, \gamma=0.2$, and $\delta=$ (a) 0.001 , (b) 0.003 , (c) 0.008 .

\section{ACKNOWLEDGMENTS}

This work was partly supported by Yazaki Memorial Foundation for Science and Technology.

\section{REFERENCES}

[1] N. Platt, E.A. Spiegel and C. Tresser, "On-Off Intermittency: A Mechanism for Bursting," Phys. Rev. Lett., vol. 70, no. 3, pp. 279-282, 1993.

[2] P. Ashwin, J. Buescu and I. Stewart, "Bubbling of Attractors and Synchronization of Chaotic Oscillators," Phys. Lett. A,193, pp. 126-139, 1994.

[3] E. Ott and J.C. Sommerer, "Blowout Bifurcations: the Occurrence of Riddled Basins and On-Off Intermittency," Phys. Lett. A, 199, pp. 39-47, 1994.

[4] Y. Nishio and A. Ushida, "Spatio-Temporal Chaos in Simple Coupled Chaotic Circuits," IEEE Trans. Circuits Syst. I, vol. 42, no. 10, pp. 678-686, 1995.

[5] N.F. Rul'kov and M.M. Sushchik, "Robustness of Synchronized Chaotic Oscillations," Int. J. Bifurcation and Chaos, vol. 7, no. 3, pp. 625-643, 1997.

[6] M. Wada, Y. Nishio and A. Ushida, "Analysis of Bifurcation Phenomena in Two Chaotic Circuits Coupled by an Inductor," IEICE Trans. Fundamentals, vol. E80-A, no. 5, pp. 869-875, 1997.

[7] Y. Nishio and A. Ushida, "Chaotic Wandering and its Analysis in Simple Coupled Chaotic Circuits," IEICE Trans. Fundamentals, vol. E85-A, no. 1, pp. 248-255, 2002.

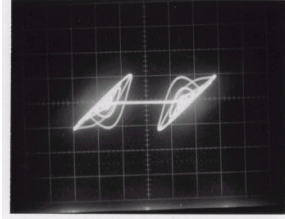

(1a)

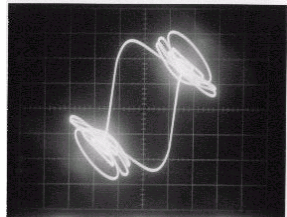

(1b)

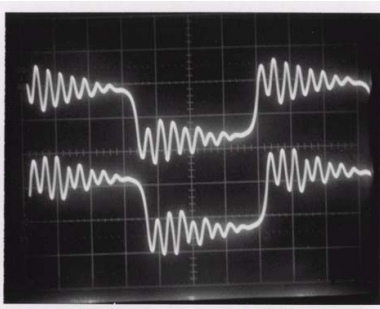

(1c)

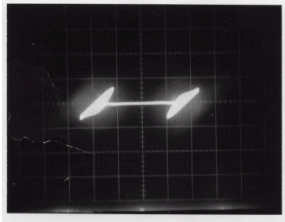

(2a)

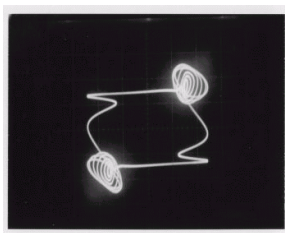

(2b)

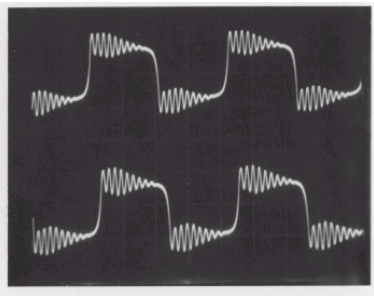

$(2 c)$

Fig. 5. State transition phenomenon for different coupling parameters (circuit experimental result). $L_{1}=9.93 \mathrm{mH}, C_{1}=32.8 \mathrm{nF}$, and $C_{2}=49.5 \mathrm{nF}$. (1) $L_{2}=$ $800 \mathrm{mH}$ and $g=626 \mathrm{mS}$. (2) $L_{2}=1.2 \mathrm{H}$ and $g=495 \mathrm{mS}$. (a) Attractor on $v_{11}$ $v_{12}$ plane. Horizontal and vertical: $1 \mathrm{~V} /$ div. (b) Attractor on $v_{11}-v_{21}$ plane. Horizontal and vertical: $1 \mathrm{~V} / \mathrm{div}$. (c) Time waveform $v_{11}$ and $v_{21}$. Horizontal $0.5 \mathrm{~ms} / \mathrm{div}$ for (1) and $1.0 \mathrm{~ms} / \mathrm{div}$ for (2), and vertical: $2 \mathrm{~V} / \mathrm{div}$.

[8] G. Abramson,V.M. Kenkre and A.R. Bishop, "Analytic Solutions for Nonlinear Waves in Coupled Reacting Systems," Physica A: Statistical Mecanics and its Applications, vol. 305, no. 3-4, pp. 427-436, 2002.

[9] I. Belykh, M. Hasler, M. Lauret and H. Nijmeijer, "Synchronization and Graph Topology," Int. J. Bifurcation and Chaos, vol. 15, no. 11, pp. 3423-3433, 2005.

[10] Y. Uchitani, R. Imabayashi and Y. Nishio, "State Transition Phenomenon in Cross-Coupled Chaotic Circuits," Proceedings of NOLTA'07, pp. 397-400, 2007.

[11] M. Shinriki, M. Yamamoto and S. Mori, "Multimode Oscillations in a Modified van der Pol Oscillator Containing a Positive Nonlinear Conductance," Proc. IEEE, vol. 69, pp. 394-395, 1981.

[12] N. Inaba, T. Saito and S. Mori, "Chaotic Phenomena in a Circuit with a Negative Resistance and an Ideal Switch of Diodes," Trans. of IEICE, vol. E70, no. 8, pp. 744-754, 1987. 


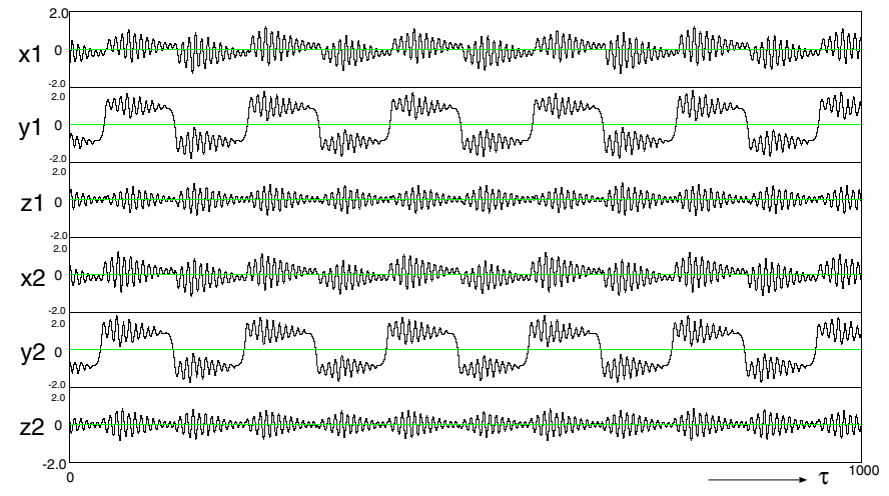

Fig. 6. Time waveforms around transition. $\alpha=1.5, \beta=5.0, \gamma=0.2$, and $\delta=0.005$.

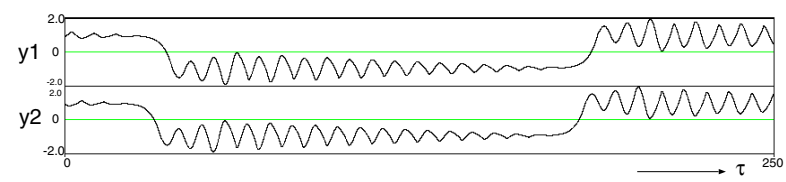

Fig. 7. Magnification of the time waveform around transition. $\alpha=1.5$, $\beta=5.0, \gamma=0.2$, and $\delta=0.003$.
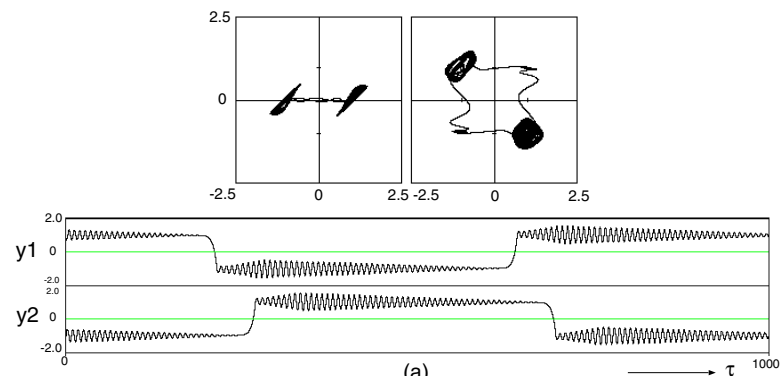

(a)
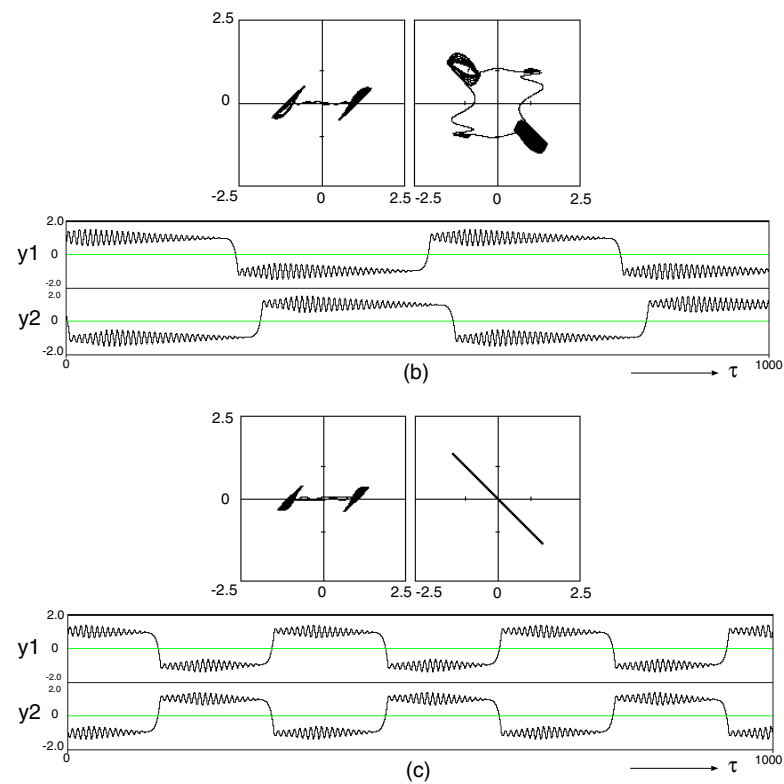

(c)

Fig. 8. Anti-phase state transition (computer calculated result). $\alpha=2.0$ $\beta=4.0, \gamma=0.1$, and (a) $\delta=0.0005$, (b) $\delta=0.0008$, (c) $\delta=0.0014$.

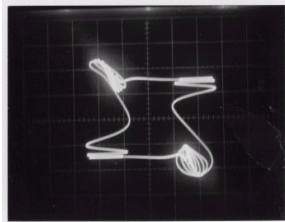

(a) (b)

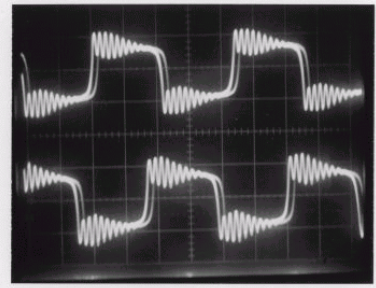

Fig. 9. Anti-phase state transition (circuit experimental result). $L_{1}=9.93 \mathrm{mH}$, $L_{2}=1.2 \mathrm{H}, C_{1}=32.8 \mathrm{nF}, C_{2}=49.5 \mathrm{nF}$, and $g=495 \mathrm{mS}$. (a) Attractor on $v_{11}-v_{21}$ plane. Horizontal and vertical: $1 \mathrm{~V} /$ div. (b) Time waveform $v_{11}$ and $v_{21}$ Horizontal $1.0 \mathrm{~ms} / \mathrm{div}$ and vertical: $2 \mathrm{~V} / \mathrm{div}$
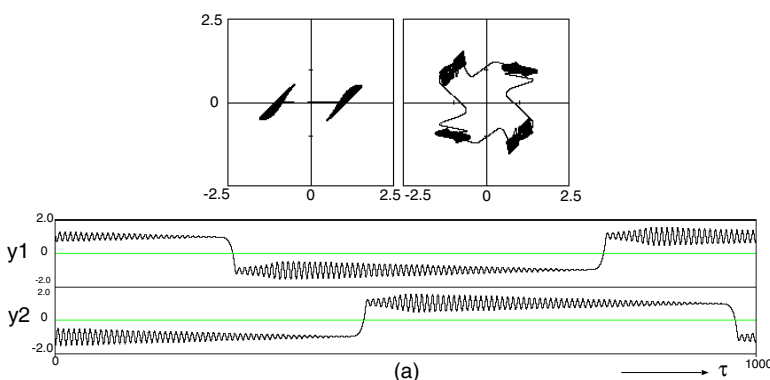

(a)
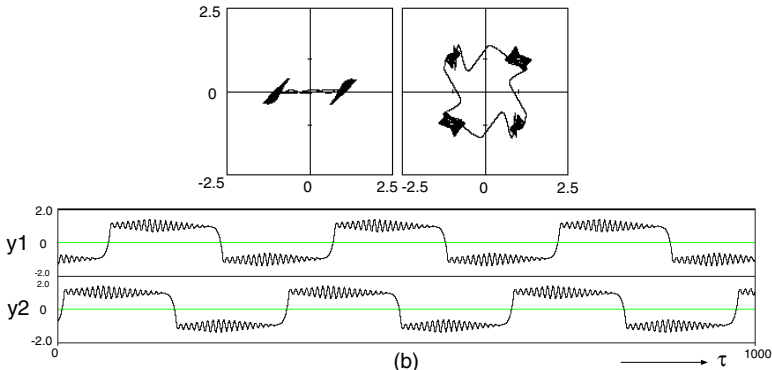

Fig. 10. Quadrature-phase state transition (computer calculated result). $\alpha=$ $2.0, \beta=4.0, \gamma=0.1$, and (a) $\delta=0.0004$, (b) $\delta=0.0014$.

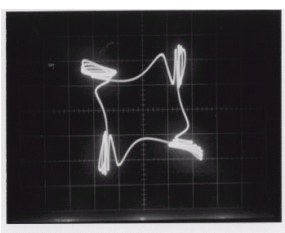

(a)

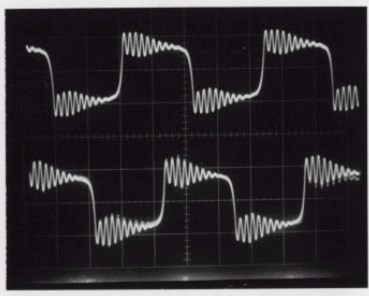

(b)
Fig. 11. Quadrature-phase state transition (circuit experimental result). $L_{1}$ $=9.93 \mathrm{mH}, L_{2}=1.2 \mathrm{H}, C_{1}=32.8 \mathrm{nF}, C_{2}=49.5 \mathrm{nF}$, and $g=495 \mathrm{mS}$. (a) Attractor on $v_{11}-v_{21}$ plane. Horizontal and vertical: 1 V/div. (b) Time waveform $v_{11}$ and $v_{21}$. Horizontal $1.0 \mathrm{~ms} / \mathrm{div}$ and vertical: $2 \mathrm{~V} /$ div. 